\title{
On the variational theory of cell-membrane equilibria
}

\author{
DAVID STEIGMANN ${ }^{\dagger}$ \\ Department of Mechanical Engineering, University of California, \\ Berkeley, CA 94720, USA \\ EVELINE BAESU ${ }^{*}$ \\ Department of Engineering Mechanics, University of Nebraska-Lincoln, \\ Lincoln, NE 68588, USA \\ ROBERT E. RUDD ${ }^{\S}$ AND JIM BELAK ${ }^{\text {II }}$ \\ Condensed Matter Physics Division, Lawrence Livermore National Laboratory, \\ Livermore, CA 94551, USA \\ AND \\ MIKE MCELFRESH" \\ Materials Research Institute, Lawrence Livermore National Laboratory, \\ Livermore, CA 94551, USA
}

[Received 28 June 2002 and in revised form 17 June 2003]

\begin{abstract}
The equivalence of two approaches to the variational theory of cell-membrane equilibria which have been proposed in the literature is demonstrated. Both assume a constraint on surface area, global in one formulation and local in the alternative, in accordance with measurements which reveal negligible surface dilation in the presence of membrane deformation. We thus address a potential controversy in the mathematical modeling of an important problem in biophysics.
\end{abstract}

Keywords: Cell-membrane equilibria; shape equation; stability.

\section{Introduction}

Mathematical models of the mechanics of phospholipid cell membranes have been shown to be predictive with respect to the observed shapes of red blood cells. Typically, the equations describing equilibrium configurations are obtained from an energy functional which accounts for the bending elasticity of the membrane enclosing the cell. The differential equation to be solved for the shape of the membrane, which we refer to as the shape equation, is then obtained by applying standard variational procedures in the presence of global constraints on the enclosed volume and the total surface area. The first constraint is intended to account for the incompressibility of the fluid medium enclosed by the membrane together with the impermeability of the membrane itself; the second

\footnotetext{
†Email: steigman@me.berkeley.edu

‡Email: ebaesu@unlserve.unl.edu

$\S$ Email: robert.rudd@1lnl.gov

II Email: belak1@1lnl.gov

"Email: mcelfresh@1lnl.gov
} 
for the significant energetic penalty attending surface dilation. These constraints are appropriate idealizations of the observed phenomenology over time scales associated with typical experiments [5]. The approach described here has been advanced in [12] for general equilibrium membrane shapes, and in [4] for a class of axisymmetric shapes. In the latter setting, the model has been shown to generate remarkable agreement with observations on cell-membrane equilibria. An extension of the axisymmetric problem to dynamic, acceleration-free motions has been analyzed in [13], where a thorough discussion of phenomenology and a comprehensive bibliography may also be found.

In another approach to this subject, the equilibrium equations are recovered from those for a general elastic surface with bending and stretching resistance by incorporating a local energy density which reflects the two-dimensional fluidity of the phospholipid membrane [1, 8, 14]. In this approach the final equation for the shape is recovered by making use of the tangential component of the equilibrium operator but with the global area constraint replaced by a local constraint, whereas the tangential equation does not arise in the first approach. Remarkably, the two approaches yield precisely the same shape equation. Mathematically, the difference between them is due to the fact that the relevant Lagrange multiplier is uniform in the first approach and a function of surface coordinates in the second. Their equivalence would thus appear to be unexpected at the outset. Our purpose in this note is to demonstrate this equivalence and to thereby resolve a potential controversy in the theory of cell-membrane mechanics. In so doing we derive variational formulae that are at once more concise and more general than those given elsewhere (e.g. [12]). We also recover some important formulae which were given without derivation in [10].

Notation and terminology used here are standard in the differential geometry of surfaces (see [17]). Thus, Greek indices take values in $\{1,2\}$ and are always summed if repeated. Subscripts preceded by commas refer to partial derivatives with respect to surface coordinates $\theta^{\alpha}$ and subscripted semi-colons are used to denote covariant derivatives.

\section{Energy functional}

The model of the mechanical response of the membrane is embodied in a strain-energy per unit area of the membrane surface $\omega$ given by the function $W(H, K)$, where $H$ is the mean curvature of the surface and $K$ is the Gaussian curvature. These are defined by

$$
H=\frac{1}{2} a^{\alpha \beta} b_{\alpha \beta} \quad \text { and } \quad K=\frac{1}{2} \varepsilon^{\alpha \beta} \varepsilon^{\lambda \mu} b_{\alpha \lambda} b_{\beta \mu},
$$

where $a^{\alpha \beta}=a_{\alpha \beta}^{-1}$ is the dual metric, the inverse of the matrix $a_{\alpha \beta}$ of metric components, $\varepsilon^{\alpha \beta}=$ $a^{-1 / 2} e^{\alpha \beta}$ is the permutation tensor density with $a=\operatorname{det}\left(a_{\alpha \beta}\right), e^{12}=-e^{21}=1, e^{11}=e^{22}=0$, and $b_{\alpha \beta}$ are the coefficients of the second fundamental form. The latter are the covariant components of the surface curvature. The contravariant adjugate of $b_{\alpha \beta}$ is given by

$$
\tilde{b}^{\alpha \beta}=\varepsilon^{\alpha \lambda} \varepsilon^{\beta \gamma} b_{\lambda \gamma}
$$

and satisfies

$$
b_{\mu}^{\beta} \tilde{b}^{\mu \alpha}=K a^{\beta \alpha},
$$

where $b_{\beta}^{\alpha}$ are the mixed components of the curvature. The latter figures in the Gauss and Weingarten equations

$$
\mathbf{a}_{\alpha ; \beta}=b_{\alpha \beta} \mathbf{n} \quad \text { and } \quad \mathbf{n}_{, \alpha}=-b_{\alpha}^{\beta} \mathbf{a}_{\beta},
$$


respectively. Here, $\mathbf{a}_{\alpha}=\mathbf{r}_{, \alpha}$ are the tangent vectors induced by the parametrization $\mathbf{r}\left(\theta^{\alpha}\right)$, the position in 3-space of a point on $\omega$ with coordinates $\theta^{\alpha}$, and $\mathbf{n}\left(\theta^{\alpha}\right)$ is the local surface orientation $\left(=\frac{1}{2} \varepsilon^{\alpha \beta} \mathbf{a}_{\alpha} \times \mathbf{a}_{\beta}\right)$. The $\mathbf{a}_{\alpha}$ are related to the metric by $a_{\alpha \beta}=\mathbf{a}_{\alpha} \cdot \mathbf{a}_{\beta}$, where the dot refers to the Euclidean inner product on the translation space of the enveloping three-dimensional Euclidean point space.

Equilibrium configurations are by definition those which render stationary the potential energy functional [1, 14]

$$
E=\int_{\omega}[W(H, K)-\gamma] \mathrm{d} a-p V(\omega)
$$

where $V(\omega)$ is the volume enclosed by $\omega$ and $\gamma\left(\theta^{\alpha}\right)$ and $p$ are Lagrange multipliers associated with area and volume, respectively. The first multiplier is a function defined on $\omega$ in accordance with the prescription of a constraint on local area (equivalently, local mass density); the second is a constant associated with the assignment of a global constraint on enclosed volume. The local area constraint is an idealization of the empirical observation that cell membranes are very stiff against local areal dilation. Deformations which entail dilation are thus energetically disfavored unless required by subsidiary conditions or boundary data [5]. However, in this work the surfaces considered are closed and such conditions are not relevant. The constraint on volume is global rather than local because the domain of the problem is the surface $\omega$; that is, the volume $V$ is expressible as an integral with domain $\omega$ and we do not account explicitly for the local properties of the fluid medium contained in $V$.

Several observations regarding (5) may be made before proceeding. First, Lagrange multipliers associated with local constraints are in general functions of the surface coordinates [7]. In principle, the Euler equations yield differential equations which specify the manner in which the multiplier may vary with coordinates in equilibrium states. This is similar to the problem of generating the constraint pressure in a three-dimensional elastic solid subject to a local constraint on volume [6]. A local constraint effectively adds a differential equation to the system. In the general context, the augmented system would be overdetermined unless the associated Lagrange multiplier were permitted to be a function of coordinates. One of our objectives here is to show that, these observations notwithstanding, the multiplier associated with the local area constraint is in fact constant in equilibrium. The result is readily understood via an analogy discussed in Section 4.

Second, the energy $W$ is seen to be an isotropic function of the curvature tensor on $\omega$. This form emerges as the canonical representation for the energy of an elastic surface which is distorted relative to some fixed reference surface, in accordance with the nonlinear theory of elastic shells, provided that the symmetry group of the shell is taken to be the unimodular group of local areapreserving transformations [14]. This concept is the natural extension to two dimensions of the definition of fluidity introduced by Noll [11] in the three-dimensional context. It is not equivalent to the notion of isotropic symmetry relative to the reference surface; the latter is associated with the mechanical response of certain solids. In the present setting, the completeness of the canonical form $W(H, K)$ for fluid films is proved in [15].

Lastly, the local energy is evidently insensitive to area-preserving flows of the fluid on the surface $\omega$. These are associated with acceleration-free swirling patterns observed on macroscopic soap bubbles which leave the surface shape invariant [16]. 


\section{Local variational derivatives}

To compute the variational derivative (or Gateaux differential) of the energy functional it is necessary to have explicit formulas for the variational derivatives of $H$ and $K$. We denote variational differentiation by a superposed dot. This is simply the derivative of the considered quantity with respect to a parameter which identifies configurations of the surface. The dot notation refers to the value of the derivative at a fixed value of the parameter, zero say, which we use to identify quantities associated with the particular equilibrium state considered.

The derivatives $\dot{H}$ and $\dot{K}$ are induced by the virtual displacement $\mathbf{u}\left(\theta^{\alpha}\right)=\dot{\mathbf{r}}$. The usual product rule and equations (1) yield

$$
2 \dot{H}=\dot{a}^{\alpha \beta} b_{\alpha \beta}+a^{\alpha \beta} \dot{b}_{\alpha \beta} \quad \text { and } \quad 2 \dot{K}=e^{\alpha \beta} e^{\lambda \mu}\left[\frac{1}{a}\left(\dot{b}_{\alpha \lambda} b_{\beta \mu}+b_{\alpha \lambda} \dot{b}_{\beta \mu}\right)-\frac{\dot{a}}{a^{2}} b_{\alpha \lambda} b_{\beta \mu}\right] .
$$

In [14] it is shown that

$$
\dot{a}_{\alpha \beta}=\mathbf{a}_{\alpha} \cdot \mathbf{u}_{, \beta}+\mathbf{a}_{\beta} \cdot \mathbf{u}_{, \alpha} \quad \text { and } \quad \dot{b}_{\alpha \beta}=\mathbf{n} \cdot \mathbf{u}_{; \alpha \beta},
$$

where $\mathbf{u}_{; \alpha \beta}$ is the second covariant derivative of $\mathbf{u}$. We therefore seek to represent $(6)$ in terms of 77. To this end we evaluate the variational derivative of the identity $a^{\alpha \lambda} a_{\lambda \beta}=\delta_{\beta}^{\alpha}$, where $\delta_{\beta}^{\alpha}$ is the Kronecker delta (equal to unity if $\alpha=\beta$ and to zero otherwise), to obtain

$$
\dot{a}^{\alpha \beta}=-a^{\alpha \gamma} a^{\beta \lambda} \dot{a}_{\gamma \lambda} .
$$

The definition of the scalar density $a$ further implies that

$$
\dot{a} / a=a^{\alpha \beta} \dot{a}_{\alpha \beta} .
$$

We will also make use of the scalar

$$
J=\sqrt{a / A},
$$

where $A$ is the value of $a$ on a fixed reference surface $\Omega$. The variational derivative of $A$ vanishes, yielding

$$
\dot{J} / J=\frac{1}{2} a^{\alpha \beta} \dot{a}_{\alpha \beta} .
$$

Combining these results with (8), substituting into (6), and using the dual metric to raise indices, we derive

$$
2 \dot{H}=a^{\alpha \beta} \dot{b}_{\alpha \beta}-b^{\alpha \beta} \dot{a}_{\alpha \beta} \quad \text { and } \quad \dot{K}=-K a^{\alpha \beta} \dot{a}_{\alpha \beta}+\tilde{b}^{\alpha \beta} \dot{b}_{\alpha \beta} .
$$

These in turn may be combined with (7) to obtain expressions involving the virtual displacement $\mathbf{u}$. The latter consists of components parallel and perpendicular to the tangent planes of the equilibrium surface $\omega$ which are associated with tangential and normal variations, respectively.

\section{(a) Tangential variations}

For tangential variations the virtual displacement $\mathbf{u}$ takes the form

$$
\mathbf{u}=u^{\lambda} \mathbf{a}_{\lambda}
$$

Then

$$
\mathbf{u}_{, \alpha}=u_{; \alpha}^{\beta} \mathbf{a}_{\beta}+u^{\lambda} b_{\lambda \alpha} \mathbf{n},
$$


where

$$
u_{; \alpha}^{\beta}=u_{, \alpha}^{\beta}+u^{\lambda} \Gamma_{\lambda \alpha}^{\beta},
$$

and $\Gamma_{\beta \lambda}^{\alpha}$ are the Riemannian connection coefficients induced by the coordinates on $\omega$. This yields

$$
\dot{a}_{\alpha \beta}=u_{\alpha ; \beta}+u_{\beta ; \alpha} .
$$

Further,

$$
\dot{b}_{\alpha \beta}=\left(\mathbf{n} \cdot \mathbf{u}_{, \alpha}\right)_{; \beta}-\mathbf{n}_{, \beta} \cdot \mathbf{u}_{, \alpha}=\left(u^{\lambda} b_{\lambda \alpha}\right)_{; \beta}+b_{\beta}^{\lambda} \mathbf{a}_{\lambda} \cdot \mathbf{u}_{, \alpha} .
$$

We thus derive

$$
\dot{b}_{\alpha \beta}=u_{; \alpha}^{\lambda} b_{\lambda \beta}+u_{; \beta}^{\lambda} b_{\lambda \alpha}+u^{\lambda} b_{\lambda \alpha ; \beta}
$$

Using the fact that the metric is covariant-constant we obtain

$$
a^{\alpha \beta} \dot{b}_{\alpha \beta}=2 b_{\beta}^{\alpha} u_{; \alpha}^{\beta}+u^{\alpha} b_{\alpha ; \beta}^{\beta}
$$

which we combine with

$$
b^{\alpha \beta} \dot{a}_{\alpha \beta}=2 b^{\alpha \beta} u_{\alpha ; \beta}=2 b_{\beta}^{\alpha} u_{; \alpha}^{\beta}
$$

to find

$$
2 \dot{H}=u^{\alpha} b_{\alpha ; \beta}^{\beta} .
$$

Expanding and using the Mainardi-Codazzi equations

$$
b_{\alpha ; \beta}^{\lambda}=b_{\beta ; \alpha}^{\lambda}
$$

finally yields

$$
\dot{H}=u^{\alpha} H_{, \alpha} .
$$

This is the directional derivative of the equilibrium $H$-field along $\mathbf{u}$.

To obtain an expression for $\dot{K}$ we use (7) and 16 to derive

$$
a^{\alpha \beta} \dot{a}_{\alpha \beta}=2 u_{; \alpha}^{\alpha}
$$

and

$$
\tilde{b}^{\alpha \beta} \dot{b}_{\alpha \beta}=\left(u_{; \alpha}^{\lambda} b_{\lambda \beta}+u_{; \beta}^{\lambda} b_{\lambda \alpha}\right) \tilde{b}^{\alpha \beta}+u^{\lambda}\left(b_{\lambda \alpha} \tilde{b}^{\alpha \beta}\right)_{; \beta},
$$

where, in the last term, we have used the fact that the adjugate of the surface curvature is divergencefree [14]:

$$
\tilde{b}_{; \beta}^{\alpha \beta}=0 .
$$

To reduce 25 to a manageable form we use 3 to obtain $b_{\lambda \alpha} \tilde{b}^{\alpha \beta}=\delta_{\lambda}^{\beta} K$ and $\left(b_{\lambda \alpha} \tilde{b}^{\alpha \beta}\right)_{; \beta}=K$, . Substitution of (24) and (25) into (12) then furnishes the counterpart of (23):

$$
\dot{K}=u^{\alpha} K_{, \alpha}
$$

Finally, (11) and (16) combine to yield

$$
\dot{J} / J=u_{; \alpha}^{\alpha}
$$

the surface divergence of $\mathbf{u}$. 
(b) Normal variations

Normal variations are associated with virtual displacements of the form

$$
\mathbf{u}=u\left(\theta^{\alpha}\right) \mathbf{n} .
$$

Then

$$
\mathbf{u}_{, \alpha}=u_{, \alpha} \mathbf{n}-u b_{\alpha}^{\beta} \mathbf{a}_{\beta} .
$$

This gives

$$
\dot{a}_{\alpha \beta}=-2 u b_{\alpha \beta} \quad \text { and } \quad \dot{b}_{\alpha \beta}=u_{; \alpha \beta}-u b_{\alpha \lambda} b_{\beta}^{\lambda} .
$$

In particular,

$$
b^{\alpha \beta} \dot{a}_{\alpha \beta}=-2 u b^{\alpha \beta} b_{\alpha \beta} .
$$

We use the Cayley-Hamilton theorem in the form

$$
b^{\alpha \beta}=2 H a^{\alpha \beta}-\tilde{b}^{\alpha \beta},
$$

together with (3) and (32), to derive

$$
b^{\alpha \beta} \dot{a}_{\alpha \beta}=-2 u\left(4 H^{2}-2 K\right) .
$$

Further, (1), (3) and (31) may be used to show that

$$
a^{\alpha \beta} \dot{b}_{\alpha \beta}=a^{\alpha \beta} u_{; \alpha \beta}-u\left(4 H^{2}-2 K\right), \quad a^{\alpha \beta} \dot{a}_{\alpha \beta}=-4 u H,
$$

and

$$
\tilde{b}^{\alpha \beta} \dot{b}_{\alpha \beta}=\tilde{b}^{\alpha \beta} u ; \alpha \beta-2 K H u .
$$

These finally yield

$$
2 \dot{H}=\Delta u+u\left(4 H^{2}-2 K\right), \quad \dot{K}=2 K H u+\left(\tilde{b}^{\alpha \beta} u_{, \alpha}\right)_{; \beta}
$$

and

$$
\dot{J} / J=-2 H u,
$$

where $\Delta(\cdot)=(\cdot)_{; \alpha \beta} a^{\alpha \beta}$ is the surface Laplacian.

\section{Stationarity and the shape equation}

Equilibrium states render the total energy (5) stationary against geometric variations. To evaluate the variational derivative of $E$ we must account for the fact that the surface $\omega$ is not fixed. To this end we use the fact that the area measure on $\omega$ is $\mathrm{d} a=\sqrt{a} \mathrm{~d} \theta^{1} \mathrm{~d} \theta^{2}=J \mathrm{~d} A$, where $J$ is defined in (10) and $\mathrm{d} A=\sqrt{A} \mathrm{~d} \theta^{1} \mathrm{~d} \theta^{2}$ is the area measure on the fixed surface $\Omega$. We evaluate the derivative and then transform back to the domain $\omega$. Thus,

$$
\dot{E}=\int_{\omega} \dot{W} \mathrm{~d} a+\int_{\omega}(W-\gamma) \dot{J} / J \mathrm{~d} a-p \dot{V}
$$

Here we have conformed to the convention that the variational derivatives of the Lagrange multipliers vanish. Alternatively, the requirement that $\dot{E}$ vanish for arbitrary variations of the 
multipliers simply returns the constraints and so the variational derivatives of the multipliers need not be made explicit.

To express $\dot{V}$ in terms of $\mathbf{u}$ we extend the latter smoothly as a function in $V \cup \omega$ and write

$$
\dot{V}=\int_{V} \operatorname{div} \mathbf{u} \mathrm{d} x=\int_{\omega} \mathbf{u} \cdot \mathbf{n} \mathrm{d} a=\int_{\omega} u \mathrm{~d} a .
$$

This vanishes for purely tangential variations, for which we use

$$
\dot{W}=W_{H} \dot{H}+W_{K} \dot{K}
$$

with (23) and (27) to derive

$$
\dot{E}=\int_{\omega} u^{\alpha} W_{, \alpha} \mathrm{d} a+\int_{\omega}(W-\gamma) u_{; \alpha}^{\alpha} \mathrm{d} a=\int_{\omega}\left[(W-\gamma) u^{\alpha}\right]_{; \alpha} \mathrm{d} a+\int_{\omega} u^{\alpha} \gamma_{, \alpha} \mathrm{d} a,
$$

where $W$, regarded as a function of the $\theta^{\alpha}$, is the equilibrium distribution of strain energy.

For a closed surface on which the scalar density $a\left(\theta^{\alpha}\right)=\operatorname{det}\left(a_{\alpha \beta}\right)$ is continuous, the surface integral of the divergence of any smooth function vanishes by virtue of Stokes' theorem. Then, the first integral after the right-most equality in (42) vanishes, so that $E$ is stationary under tangential variations if and only if

$$
\int_{\omega} u^{\alpha} \gamma_{, \alpha} \mathrm{d} a=0
$$

for all choices of the $u^{\alpha}$. Choosing $u^{\alpha}=a^{\alpha \beta} \gamma_{, \beta}$ and invoking the positive-definiteness of the metric yields $\gamma_{, \alpha}=0$, or

$$
\gamma\left(\theta^{\beta}\right)=-\lambda,
$$

a constant function of surface coordinates. The sign is inserted to conform to the notation of [12]. This is the surface analogue of the well-known result that the pressure distribution in a threedimensional fluid, in equilibrium without body forces, is uniform. The latter result would have emerged in the present context also, had a local volume constraint on the enclosed fluid been imposed.

Conversely, since $\gamma$ is independent of the $u^{\alpha}$ the result is necessary and sufficient for $E$ to be stationary under all tangential variations. In fact, since $\gamma$ may be assumed to have zero variational derivative for all virtual displacements, it is equal to its equilibrium value as given by (44). Substituting into (5), we obtain the functional

$$
E^{\prime}=\int_{\omega} W(H, K) \mathrm{d} a+\lambda S(\omega)-p V(\omega),
$$

where $S(\omega)$ is the total surface area of $\omega$ and $W$ is no longer restricted to be the equilibrium strain energy. This coincides with the total energy as defined in [10, 12]. The constant $\lambda$ is formally identical to a Lagrange multiplier associated a constraint on total surface area. Following our previous development, it is then clear that $E^{\prime}$ is nontrivial only for purely normal variations.

For normal variations $(37)$ leads to

$$
\begin{aligned}
2 W_{H} \dot{H} & =u W_{H}\left(4 H^{2}-2 K\right)+\left(W_{H} a^{\alpha \beta} u_{, \alpha}\right)_{; \beta}-\left(W_{H}\right)_{, \beta} a^{\alpha \beta} u_{, \alpha} \\
& =u\left[\left(W_{H}\right)_{; \beta \alpha} a^{\beta \alpha}+W_{H}\left(4 H^{2}-2 K\right)\right]+\left(W_{H} a^{\alpha \beta} u_{, \alpha}\right)_{; \beta}-\left[\left(W_{H}\right)_{, \beta} a^{\alpha \beta} u\right]_{; \alpha}
\end{aligned}
$$


and

$$
\begin{aligned}
W_{K} \dot{K} & =\left(2 K H W_{K}\right) u+\left(W_{K} \tilde{b}^{\alpha \beta} u_{, \alpha}\right)_{; \beta}-\left(W_{K}\right)_{, \beta} \tilde{b}^{\alpha \beta} u_{, \alpha} \\
& =u\left[\left(W_{K}\right)_{; \beta \alpha} \tilde{b}^{\beta \alpha}+2 K H W_{K}\right]+\left(W_{K} \tilde{b}^{\alpha \beta} u_{, \alpha}\right)_{; \beta}-\left[\left(W_{K}\right)_{, \beta} \tilde{b}^{\alpha \beta} u\right]_{; \alpha} .
\end{aligned}
$$

Modulo divergences which do not contribute to the variational derivative of the energy of a closed surface, we then have

$$
\dot{W}=u\left[\Delta\left(\frac{1}{2} W_{H}\right)+\left(W_{K}\right)_{; \alpha \beta} \tilde{b}^{\alpha \beta}+W_{H}\left(2 H^{2}-K\right)+2 K H W_{K}\right] .
$$

Further, in place of the second integrand in (39) we substitute

$$
(W+\lambda) \dot{J} / J=-u[2 H(W+\lambda)] .
$$

Then with 45 we find that the energy $E^{\prime}$ (or $E$ ) is stationary against normal variations if and only if the equilibrium shape equation

$$
\Delta\left(\frac{1}{2} W_{H}\right)+\left(W_{K}\right)_{; \alpha \beta} \tilde{b}^{\alpha \beta}+W_{H}\left(2 H^{2}-K\right)+2 H\left(K W_{K}-W\right)-2 H \lambda=p
$$

is satisfied. This result may also be obtained by combining equations (6.19) and (6.21) of [14]. In the absence of local bending resistance it reduces to the classical Laplace equation of capillarity which requires that the mean curvature be uniform on $\omega$.

The best known example of 50 , is [10, 12]

$$
k\left[\Delta H+2 H\left(H^{2}-K\right)\right]-2 \lambda H=p,
$$

and corresponds to the strain-energy function

$$
W=k H^{2}+\bar{k} K
$$

wherein $k$ and $\bar{k}$ are empirical constants. This special energy may be regarded as an approximation to $W$ obtained by retaining terms quadratic in a small parameter representing the ratio of film thickness - typically on the order of the length of one or two polar molecules- to the infimum over $\omega$ of the minimum principal radius of curvature [1].

Equation [51] was derived in [10] and [12] by subjecting $E^{\prime}$ to normal variation. In these works the part of the energy involving the constant $\bar{k}$ is suppressed because, according to the applicable version of the Gauss-Bonnet theorem, it contributes a term proportional to the Euler characteristic of the surface. This has vanishing variational derivative if the topology of the surface is fixed. In the general case without topological constraints, equations (52) and (50) yield (51) because the covariant derivatives of $W_{K}(=\bar{k})$ vanish. Of course, 51 remains valid if the surface has boundaries, although the argument used in [10,12] to justify the suppression of the $\bar{k}$-term is not then applicable.

The formulation described in [10, 12], based on an energy functional subject to purely normal variations under a global area constraint, is thus seen to be equivalent to the present formulation which admits arbitrary variations under a local constraint. Indeed, the restriction to normal variations alone is not natural in mechanics, and, to our knowledge, is not motivated in the literature. 


\section{Remarks}

Solution of the shape equation is complicated by the fact that the values of the parameters $\lambda$ and $p$ are not known at the outset. In practice, when attempting to solve the differential equations directly (e.g. [1,4]), these values are initialized and then the solution to [50, computed. The solution thus obtained is used to evaluate the functions $S(\omega)$ and $V(\omega)$, which may be compared to the values required by the constraints. The constants $\lambda$ and $p$ are then adjusted until the constraints are satisfied.

A numerical scheme for solving the problem of axisymmetric Willmore flow has been presented in [9]. This is essentially a dynamical counterpart of (51) in which $\lambda, p$ and the acceleration of the film are suppressed.

Alternatively, direct minimization techniques for equilibria such as the conjugate gradient method may be used, and have proved to be a popular and effective means of solving problems involving capillary surfaces [3]. Applications of such methods to the energy functional (45) for general nonaxisymmetric surfaces are described in [12]. Since we have shown that (5) and (45) coincide only for equilibrium values of the Lagrange multiplier $\gamma$, a question naturally arises regarding the limits of distinct minimizing sequences of configurations associated with the functionals $E$ and $E^{\prime}$ separately. Since these two energies coincide in equilibrium states, it is clearly desirable that the common equilibrium value, $E_{0}$ say, should emerge as the (weak) limit of independent minimizing sequences for $E$ and $E^{\prime}$. This is the case if the energy functionals possess the property of lower semicontinuity with respect to an appropriate definition of convergence [2]. Thus, if $\left\{\omega_{n}\right\}$ and $\left\{\omega_{n}^{\prime}\right\}$ are sequences of surfaces, then we require that

$$
E_{0} \leqslant \liminf _{n \rightarrow \infty} E\left(\omega_{n}\right) \quad \text { as } n \rightarrow \infty \quad \text { and } \quad E_{0} \leqslant \liminf _{n \rightarrow \infty} E^{\prime}\left(\omega_{n}^{\prime}\right) \quad \text { as } n \rightarrow \infty
$$

whenever

$$
\omega_{n} \rightarrow \omega_{0} \text { and } \omega_{n}^{\prime} \rightarrow \omega_{0},
$$

where $\omega_{0}$ is an equilibrium surface and $E_{0}=E\left(\omega_{0}\right)=E^{\prime}\left(\omega_{0}\right)$. The notation $\rightarrow$ connotes weak or weak-* convergence.

It is known that lower semicontinuity is guaranteed by the condition of quasiconvexity [2], which is an integral inequality involving the strain-energy function $W$. A pointwise necessary condition has been derived in [14]. This is given by the local constitutive inequality

$$
F(x) \geqslant 0,
$$

where

$$
F(x)=\frac{1}{4} W_{H H}+2 x W_{H K}+x^{2} W_{K K} \quad \text { with } \quad x=\tilde{b}^{\alpha \beta} \varsigma_{\alpha} \varsigma_{\beta} \in \mathbb{R}
$$

and arbitrary $\varsigma_{\alpha} \in \mathbb{R}^{2}$. The inequality is also necessary for the existence of energy-minimizing equilibria and may thus be regarded as a necessary condition for Lyapunov stability. If $W_{K K}=0$, as in the case of (52), then, if the coefficient of $x$ in (56) is non-zero, $F(x)$ may assume negativehence inadmissible-values on a half-interval of the $x$-axis. The inequality can then be satisfied only if $W_{H K}=0$ and $W_{H H} \geqslant 0$, e.g. only if $k \geqslant 0$ in 52. If $W_{K K} \neq 0$, then $F(x) \geqslant 0$ if and only if $W_{K K}>0$ and the discriminant in 56 is nonpositive: $4 W_{K H}^{2}-W_{K K} W_{H H} \leqslant 0$.

In general lower semicontinuity alone is insufficient to secure the existence of energyminimizing surfaces [2]. Existence theory for problems of the kind considered here remains incomplete, even for surfaces with prescribed Euler characteristic (equivalently, prescribed genus). Partial results are available, however, and are summarized in [10]. 


\section{Acknowledgements}

This work was performed under the auspices of the U.S. Department of Energy by the University of California, Lawrence Livermore National Laboratory, under Contract no. W-7405-Eng-48. The authors are grateful to the referees for insightful comments leading to the improvement of the manuscript.

\section{REFERENCES}

1. Baesu, E., Rudd, R. E., Belak, J., \& McElfresh, M. Continuum modeling of cell membranes. Int. J. Non-linear Mech. (in press).

2. BALL, J. M. On the calculus of variations and sequentially weakly continuous maps. Ordinary and Partial Differential Equations, Lecture Notes in Math. 564, Springer, Berlin (1976), 13-25. Zbl 0348.49004 MR $58 \# 30605$

3. BRAKKe, K. E. The surface evolver. Experiment. Math. 1 (1992), 141-165. Zbl 0769.49033 MR 93k:53006

4. Dueling, H. J. \& Helfrich, W. The curvature elasticity of fluid membranes: a catalogue of vesicle shapes. J. Phys. 37 (1976), 1335.

5. Evans, E. A. \& SKAlaK, R. Mechanics and Thermodynamics of Biomembranes. CRC Press, Boca Raton, FL (1980).

6. Fosdick, R. L. \& MacSithigh, G. P. Minimization in incompressible nonlinear elasticity theory. J. Elasticity 16 (1986), 267-301. Zbl 0616.73026 MR 88c:73022

7. Gelfand, I. M. \& Fomin, S. V. Calculus of Variations. Prentice-Hall, New York (1963). Zbl 0127.05402 MR 28\#3353

8. Jenkins, J. T. Static equilibrium configurations of a model red blood cell. J. Math. Biol. 4 (1977), 149. Zbl 0356.92010

9. Mayer, U. F. \& Simonett, G. A numerical scheme for axisymmetric solutions of curvature-driven free boundary problems, with applications to Willmore flow. Interfaces Free Bound. 4 (2002), 89-109. Zbl 1005.65095 MR 2003g:53119

10. Nitsche, J. C. C. Boundary value problems for variational integrals involving surface curvatures. Quart. Appl. Math. 51 (1993), 363-387. Zbl 0785.35027 MR 94c:58036

11. Noll, W. A mathematical theory of the mechanical behavior of continuous media. Arch. Rat. Mech. Anal. 2 (1958), 197-226. Zbl 0083.39303 MR 21 \#4596

12. Ou-YAng. Z.-C., LiU, J.-X., \& XIE, Y.-Z. Geometric Methods in the Elastic Theory of Membranes in Liquid Crystal Phases. World Scientific, Singapore (1999). Z Zbl 0982.74002

13. Rosso, R. Asymptotic evolution of lipid vesicles. Interfaces Free Bound. 3 (2001), 345-360. Zbl 1007.3507 MR 2002f:35039

14. Steigmann, D. J. Fluid films with curvature elasticity. Arch. Rat. Mech. Anal. 150 (1999), 127-152. Zbl 0982.76012 MR 2000k:76014

15. Steigmann, D. J. Irreducible function bases for simple fluids and liquid crystal films. Z. Angew. Math. Phys. 54 (2003), 462-477.

16. Steigmann, D. J. Some inertia-free flows in soft matter. Manuscript in preparation.

17. Stoker, J. J. Differential Geometry. Wiley-Interscience, New York (1969). MR 39 \#2072 